\title{
MEDIÇÃO DO GAP ÓPTICO DE FILMES FINOS POR MEIO DAS PRÓPRIEDADES ÓPTICAS
}

\author{
J. C. A. QUEIROZ ${ }^{1}$, F. M. FERNANDES ${ }^{2}$, I. A. SOUZA ${ }^{3}$, E. J. C. SANTOS ${ }^{4}$, R. C. P. PEREIRA ${ }^{5}$, M. G. O. QUEIROZ ${ }^{6}$, T. H. \\ C. $\operatorname{COSTA}^{7}$ \\ 1,2,3,4,5,6,7 Universidade Federal do Rio Grande do Norte - Escola de Ciência e Tecnologia \\ cesaraugusto@bct.ect.ufrn.com ${ }^{1}$ \\ Submetido 12/07/2016 - Aceito 06/03/2018 \\ DOI: $10.15628 /$ holos.2018.4826
}

\section{RESUMO}

O desempenho óptico de qualquer material resulta da sua interação com uma radiação eletromagnética. Experimentalmente, através de medições de absorção óptica, é possível estimar o valor da energia fotoelétrica absorvida pelo material e obter indicações sobre o tipo de transição da banda. Neste trabalho é realizada a análise de deposições de filmes finos com $100 \%$ de argônio em substrato de vidro, depositados por magnetron sputtering, com tempos de deposição de trinta minutos e de uma hora. As constantes ópticas dos filmes foram medidas por medições de refletância óptica e transmitância. Esses valores, juntamente com micrografias obtidas no microscópio eletrônico de varredura (MEV), promovem a construção da curva que fornece a estimativa da energia de bandgap dos filmes. Os filmes finos de argônio com o tempo de deposição maior do que os outros (1 hora) são apresentados como mais condutores, devido à sua baixa energia de bandgap $\left(E_{g}^{d}=1,94 \mathrm{eV}\right)$. Os resultados sugerem que $100 \%$ de filmes de argônio podem ter um potencial considerável para serem usados como eletrodos transparentes aplicados a células solares, desde que tenham sua resposta óptica otimizada por algum elemento dopante.

PALAVRAS-CHAVE: Radiação eletromagnética, filmes finos, energia, sputtering magnetron, bandgap.

\section{MEASURING OF THE GAP OPTICAL OF THIN FILMS BY MEANS OF OPTICAL PROPERTIES}

\section{ABSTRACT}

The optical performance of any material results from its interaction with an electromagnetic radiation. Experimentally, through optical absorption measurements, it is possible to estimate the value of the photoelectric energy absorbed by the material and to acquire indications about the bandgap transition type. In this work the deposition of thin films with $100 \%$ of argon in glass substrate, deposited by magnetron sputtering, with deposition times of thirty minutes and one hour is performed. Optical constants of the films were measured by optical reflectance and transmittance measurements. These values, along with micrographs obtained in the scanning electron microscope (SEM), promote the construction of the curve that provides the estimation of the films bandgap energy. Thin films of argon with deposition time longer than the others (1 hour) are presented as more conductors due to their that $100 \%$ of argon films may have considerable potential to be used as transparent electrodes applied to solar cells as long as they have their optical response optimized by some dopant element.

KEYWORDS: electromagnetic radiation, thin films, energy, magnetron sputtering, bandgap. low bandgap energy $\left(E_{g}^{d}=1,94 \mathrm{eV}\right)$. The results suggest 


\section{INTRODUÇÃO}

Os filmes finos são materiais que podem ser classificados em metálicos, não metálicos, orgânicos ou compostos, depositados sobre outro material, por processos físicos ou químicos, com espessura que varia de 1 a $100.000 \AA$ (Scapin, 2004).

Os estudos que convergem aos filmes finos tem proporcionado a aplicação dos mesmos nas mais diversas áreas, como por exemplo: microeletrônica, telecomunicação, dispositivos e revestimentos ópticos e eletro-ópticos (Ganesh, Nair, Raut, Walsh, \& Ramakrishna, 2012; Wang, Lin, \& Yang, 2009) revestimentos de materiais e geração de energia, com diversos propósitos, dentre eles: a proteção contra a corrosão, isolamento de condutores, barreiras anti-refletoras e de difusão, miniaturização de dispositivos de alta tecnologia e até mesmo a ornamentação de objetos, dispositivos eletrocrômicos, sensores e capacitores de armazenamento de carga em circuitos integrados (Cruz, 1999; Kasap \& Capper, 2017; Liu et al., 2015; Siebentritt, 2017).

Tendo-se em vista, que as aplicações dos filmes finos englobam vários segmentos da sociedade, e que existe uma aceitação desse tipo de matéria devido ao seu valor econômico, existe a expectativa de que novos filmes proporcionem novas tecnologias ou que aperfeiçoem as já existentes. Sendo assim, se faz necessário conhecer melhor suas propriedades e compreender os fenômenos físicos que regem esses sistemas (Pazim, 2011). Tentando-se induzir uma resposta do material, a partir do conhecimento da estrutura micro e macroscópica do mesmo.

O desempenho óptico de qualquer material resulta da interação deste com a radiação eletromagnética (Ruppert, Aslan, \& Heinz, 2014). Entre os vários fenômenos que podem ser ocasionados, da interação radiação-material, destacam-se a refletância $(R)$, a transmitância $(T)$ e a absorbância $(A)$, a fim de estudar o comportamento óptico do material. Sabe-se que outros fenômenos como a refração e a atenuação da radiação, enquanto transmitida no interior do material, ocorrem. A compreensão das ocorrências a nível submicroscópico, inicia-se da interpretação das manifestações macroscópicas como a transmissão e reflexão da radiação eletromagnética nos filmes (Serway \& John Jr, 2010).

A intensidade do feixe incidente $\left(I_{0}\right)$, transmitido $\left(I_{T}\right)$ e refletido $\left(I_{R}\right)$ são parâmetros importantes dentro da técnica de análise óptica (Pehlivan, Tepehan, \& Tepehan, 2005), pois 
ajudam a determinar algumas constantes, como o coeficiente de absorção $(\alpha)$ e o coeficiente de extinção $(k)$, que são importantes para a compreensão e otimização da resposta óptica (Callister \& Rethwisch, 2011). Este trabalho tem como objetivo estimar valores de constantes ópticas e analisar a natureza do bandgap, ou banda proibida, de filmes finos de hidrogênio, depositados por sputtering magnetron com diferentes tempos de deposição. Indicando possíveis valores de energia para a ocorrência de transição entre bandas de energia para os filmes citados. Experimentalmente, através de medidas de absorção óptica, é possível estimar o valor da energia e ter indicações a respeito do tipo de transição do bandgap (Floriano, Scalvi, \& Sambrano, 2009).

Este artigo, tem como objetivo analisar o caráter condutor de filmes finos de argônio, produzidos pela técnica de deposição a plasma magnetron sputtering. As propriedades ópticas dos filmes finos foram mensuradas, por meio da medida da intensidade da radiação eletromagnética transmitida e refletida, pela estrutura dos filmes. A espessura dos filmes, obtida pela análise de microscopia eletrônica de varredura, permitiram o cálculo do coeficiente de absorção óptica $(\alpha)$, responsável pela indicação direta do valor de energia de bandgap. Os valores de energia, que permitem a transição entre bandas de energia mostra-se indicado a classificação dos filmes em semicondutores.

\section{MATERIAIS E MÉTODOS}

Neste trabalho foram preparados filmes finos de $100 \%$ argônio depositados em substrato de vidro, através da técnica de deposição física intitulada sputtering magnetron. As variáveis do processo, como fluxo de gás, pressão e temperatura foram mantidas constantes e iguais a 10 sccm, $1,5 \times 10^{-6}$ mTorr e $250^{\circ} \mathrm{C}$, respectivamente, com exceção do tempo, que variou entre 30 minutos, para a primeira deposição, e 1 hora para a segunda deposição. Formou-se dois grupos de filmes finos, apenas com a fator tempo variando. Para esse trabalho adotou-se a seguinte nomenclatura: (100\%Ar-30min) para os filmes depositados com tempo de 30 minutos e (100\%Ar1h) para os filmes depositados com tempo de 1 hora.

Utilizou-se um cubo de análises ópticas, construído e aperfeiçoado no Laboratório de Processamentos de Materiais por Plasma (LabPlasma-UFRN), com o objetivo de quantificar o feixe incidente $\left(I_{0}\right)$, transmitido $\left(I_{T}\right)$ e refletido $\left(I_{R}\right)$. Para a detecção dos feixes transmitido e refletido, o cubo apresenta configurações diferentes. A captação do feixe transmitido ocorre por meio da incidência normal do feixe $\left(I_{0}\right)$ à superfície do filme, com uma fibra óptica posicionada 
após o filme para captação do feixe $\left(I_{T}\right)$, como mostra a figura 1. Para a quantificação do feixe $\left(I_{R}\right)$, o cubo apresentou-se com uma configuração diferente, observando-se a figura 2, com a mudança de posição da fibra óptica, formando um ângulo próximo de $100^{\circ} \mathrm{com}$ o feixe $\left(I_{0}\right)$.

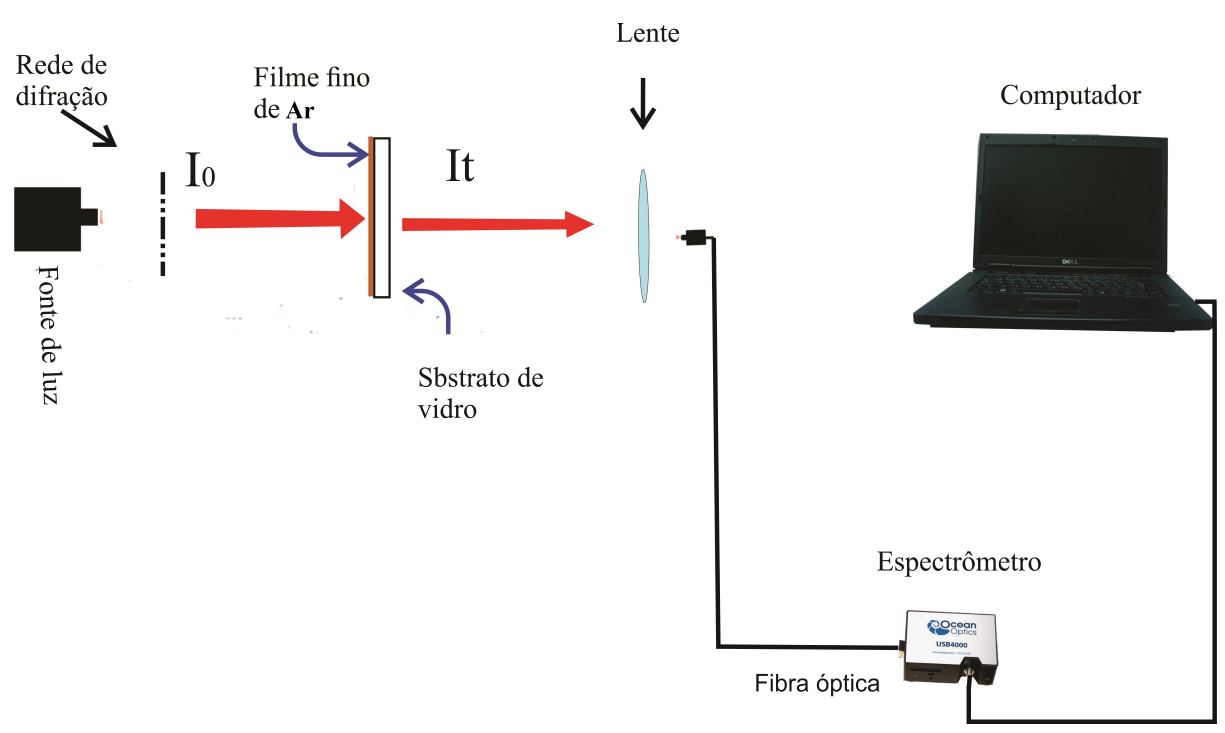

Figura 1: Esquema ilustrativo da configuração do cubo de análises ópticas para medir a transmissividade dos filmes finos de argônio.

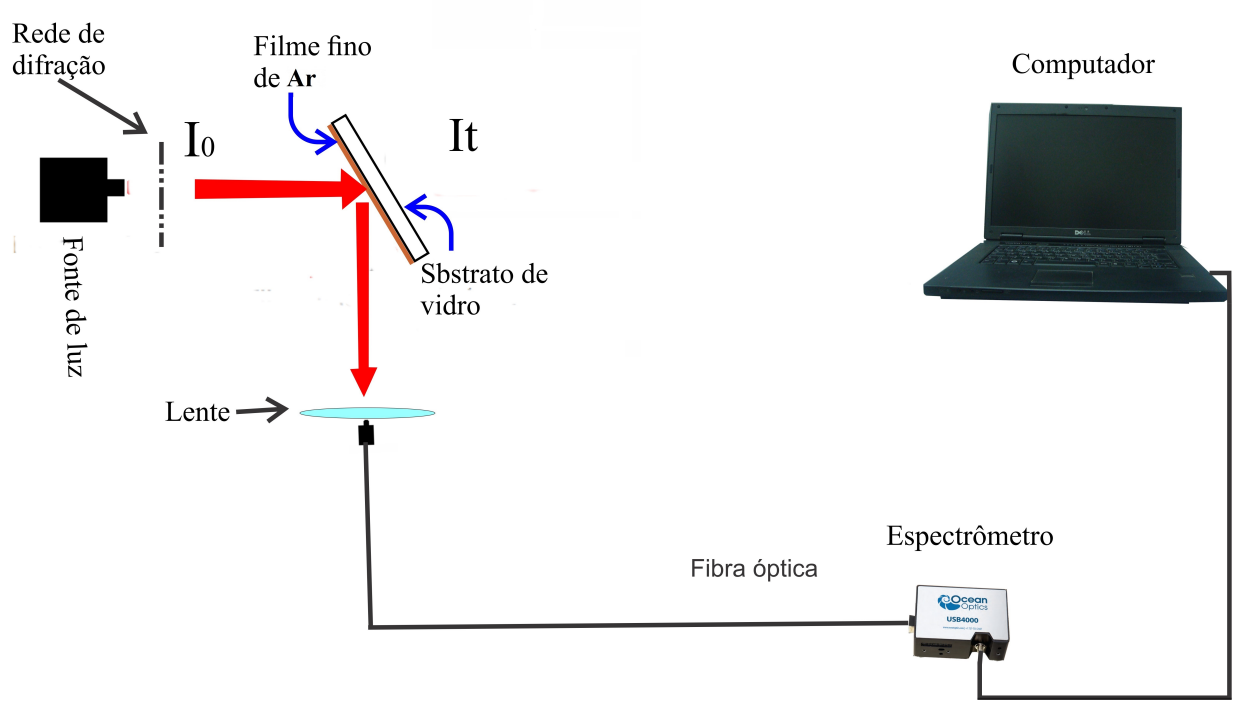

Figura 2: Esquema ilustrativo da configuração do cubo de análises ópticas para medir a refletividade dos filmes finos de argônio.

Os filmes finos de argônio, foram submetidos a uma fonte de luz monocromática, ou seja, um laser com incidência de feixe de radiação eletromagnética com comprimento de onda $(\lambda)$ fixo em $637,89 \mathrm{~nm}$. 
A captação dos feixes ocorreram com o auxílio de um Espectrômetro de Emissão Óptica, modelo USB4000 UV-VIS e resolução máxima de $1,5 \mathrm{~nm}$, em conjunto com o software SpectraSuite.

\section{RESUTADOS E DISCUSSÃO}

A refletividade $R(\lambda)$ e a transmitância $T(\lambda)$ representam, respectivamente, a fração da luz incidente refletida e transmitida na interface do filme, sendo calculadas pelas relações: $R(\lambda)=$ $I_{R} / I_{0}$ e $T(\lambda)=I_{T} / I_{0}$ (Callister \& Rethwisch, 2011). Para o filme fino de argônio (100\%Ar-30min), apresentou-se valores de refletividade em torno de $R(\lambda)=0,4$ e para o filme fino de argônio (100\%Ar-1h) obteve-se valor de refletividade calculada em torno de $R(\lambda)=0,9$.

O coeficiente de absorção ópticas $(\alpha)$ dos filmes de argônio, foram determinados pela equação 1 (Khedmi, Rabeh, \& Kanzari, 2014):

$$
\alpha=-\frac{1}{d} \ln \left[\frac{(1-R)^{2}}{T}\right]
$$

Para o filme fino (100\%Ar-30min), o valor de $\alpha$ foi de, aproximadamente, $0,8 \times 10^{-4} \mathrm{~cm}^{-1} \mathrm{e}$ para o filme (100\%Ar-1h), obteve-se um $\alpha$ correspondente a um valor próximo de $0,2 \times 10^{-4} \mathrm{~cm}^{-1}$. Associou-se esses resultados a espessura da camada dos filmes. Tendo-se para o filme com tempo de 30 minutos uma camada de espessura, $d$, de aproximadamente $1,17 \mu \mathrm{m}$ e para o filme com tempo de 1 hora, uma espessura próxima de 2,62 $\mu \mathrm{m}$, como visto na figura 3. 

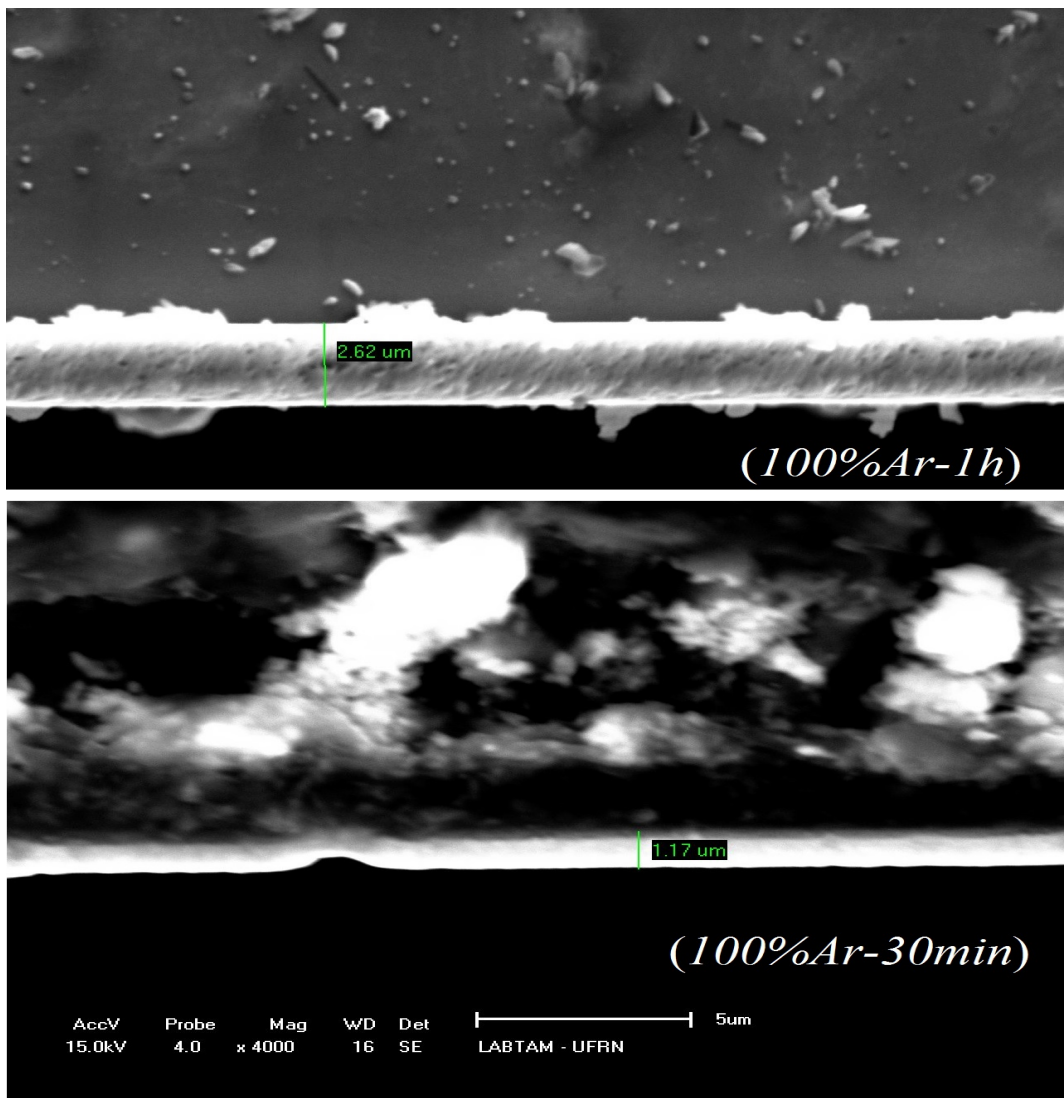

Figura 3: Micrografia eletrônica de varredura dos filmes finos de argônio com as respectivas espessuras dos filmes.

Em consulta a literatura, observou-se que filmes finos com baixa espessura apresentam baixos valores de refletividade e alta taxa de transmitância óptica, resultando em valores elevados para o coeficiente de absorção, caso inverso para os filmes de espessura alta (Bakr et al., 2011; Santos-Cruz et al., 2005).

Transições eletrônicas, entre a banda de valência (BV) e a banda de condução (BC) podem ocorrer quando a energia do fóton $(h v)$ for suficiente para superar o nível de Fermi. Essas transições eletrônicas podem ocorrer por um processo direto ou indireto, segundo (Lin, Yang, Hsi, Wang, \& Lee, 2014). A condição para que ocorra-se a absorção de um fóton (de radiação), e promova-se uma transição eletrônica em termos do comprimento de onda da radiação para um dado material, é dado pela equação 3 (Callister \& Rethwisch, 2011). Tendo-se as constantes c (velocidade da luz) e $h$ (constante de Planck) apresentando os respectivos valores e unidades, $3 \mathrm{x}$ $10^{8} \mathrm{~m} / \mathrm{s}$ e $4,13 \times 10^{-15}$ eV.s.

$$
\Delta \mathrm{E}=h v=\frac{h c}{\lambda}
$$


Estimou-se os valores de energia, necessária para promover uma transição eletrônica, de natureza direta $\left(E_{g}^{d}\right)$, por meio dos valores de absorção óptica, juntamente com as constantes apresentadas na equação 2 . Os gráficos presentes na figura 4, mostram as curvas provenientes da relação $(\alpha h v)^{2}$ vs $h v$, que de acordo com (Balakrishna \& Naik, 2017), permitem obter os valores de bandgap, com a extrapolação da porção linear da curva, em direção ao eixo x, que contém os valores de energia . Na figura 5, pode-se observa os valores de energia, indicados no eixo $h v$, que remetem a aparte da curva com coeficiente linear constante.
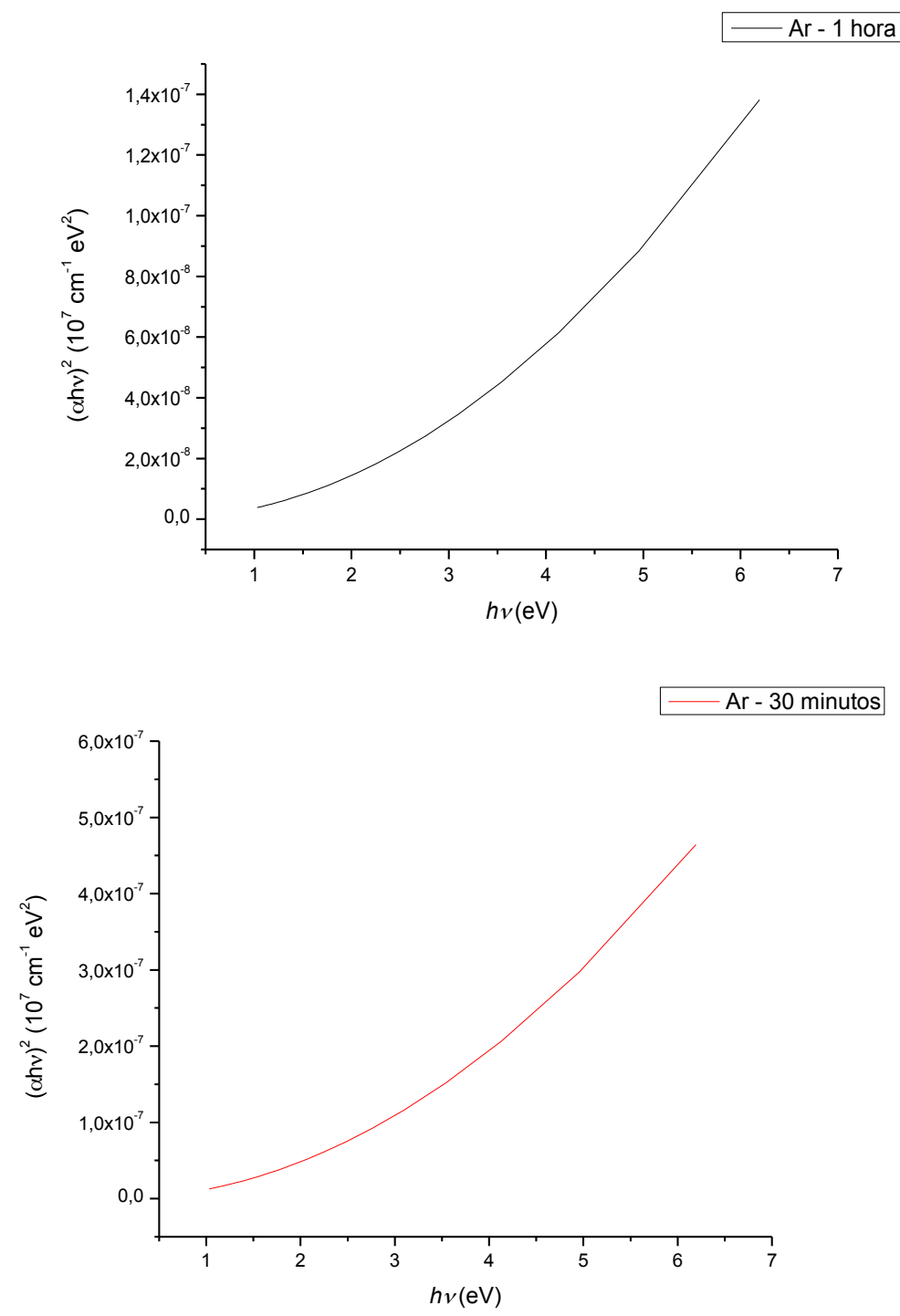

Figura 4- Gráficos de $(\alpha h v)^{2}$ em função da energia de fóton hv para os filmes de argônio com tempo de deposição de 30 minutos e 1 hora. 


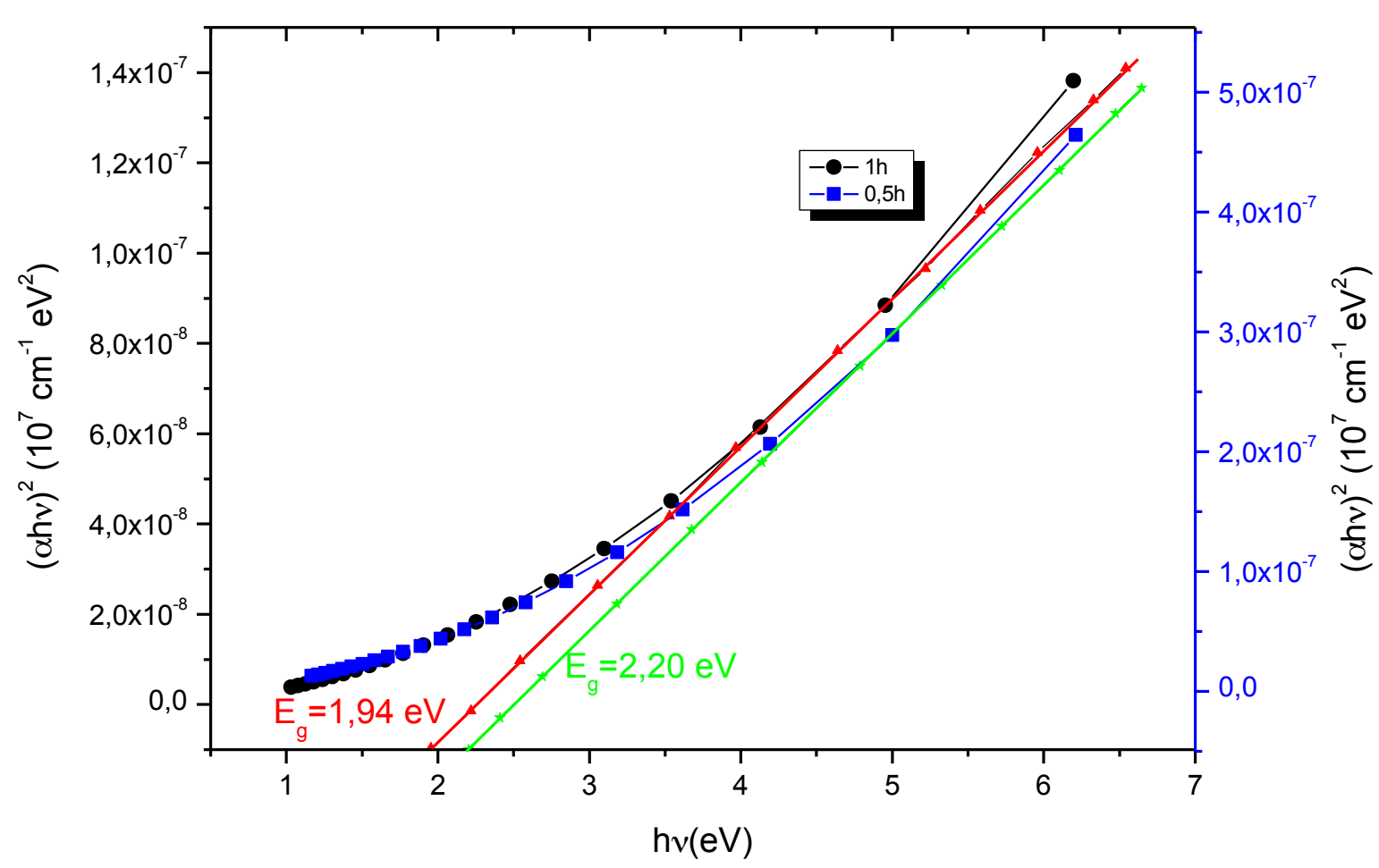

Figura 5 - Gráfico que apresenta valores de energia de banda direta para os filmes de argônio com diferentes tempos.

\section{CONCLUSÃO}

Pode-se resumir os resultados do presente estudo como se segue:

1. O filme fino de argônio (100\%Ar-30min) apresentou uma menor refletividade quando comparado ao filme de (100\%Ar-1h). Resposta, possivelmente, explicada pelo valor elevado do coeficiente de absorção óptica, que proporciona ao filme uma transmitância acentuada.

2. O filme de argônio com tempo de deposição igual a 30 minutos, apresentou uma energia de bandgap igual a $E_{g}^{d}=2,20 \mathrm{eV}$, relativamente, alta quando comparada a energia de banda direta do filme de argônio com tempo de 1 hora, $E_{g}^{d}=1,94 \mathrm{eV}$. Observou-se na literatura que, tais valores são influenciados diretamente pela refletividade $R(\lambda)$ e o coeficiente de absorção, do filme, que foram maiores para o filme de argônio com tempo de 30 minutos, justificando sua energia de transição de banda direta maior.

3. Os resultados sugerem que os filmes de $100 \%$ argônio podem apresentar um potencial considerável, para serem usados como eletrodos transparentes, aplicados em células 
solares desde que tenha sua resposta óptica seja otimizada por algum elemento dopante.

Diminuindo o valor econômico das células solares, que tem no ITO $\left(\mathrm{SnO}_{2}: \mathrm{In}\right)$, o principal

filme componente dessa tecnologia.

Diante dos resultados apresentados, pode-se concluir que o estudo relativo aos filmes finos, em especial os filmes de argônio, produzidos pela técnica de deposição a plasma, magnetron sputtering, mostra-se bastante válido, pois promove a esse tipo materiais, aplicações tanto específicas como gerais, que visam suprir a demanda da sociedade. Nesse caso, fica claro que, após a caracterização óptica dos filmes analisados no presente estudo, que os mesmos podem ser usados em superfícies de módulos fotovoltaicos, otimizando o processo de conversão de luz solar em eletricidade, em virtude de seu caráter condutor. Uma vez que, filmes finos com energia de bandgap menor que $2 \mathrm{eV}$, são classificados como materiais semicondutores. Vale salientar que as propriedades ópticas, elétricas e eletrônicas desses filmes podem ser melhoras, com o auxílio de agentes dopantes.

\section{AGRADECIMENTOS}

Os autores agradecem a Coordenação de Aperfeiçoamento de Pessoal de Nível Superior (CAPES, Brasil), a Universidade Federal do Rio Grande do Norte (UFRN) e ao Laboratório de Processamento de Materiais por Plasma (LabPlasma), pelo apoio financeiro e acesso a infraestrutura, necessária as caracterizações usadas no presente estudo.

\section{REFERÊNCIAS}

Bakr, N. A., Funde, A. M., Waman, V. S., Kamble, M. M., Hawaldar, R. R., Amalnerkar, D. P., . . . Jadkar, S. R. (2011). Determination of the optical parameters of a-Si: $\mathrm{H}$ thin films deposited by hot wire-chemical vapour deposition technique using transmission spectrum only. Pramana, 76(3), 519-531.

Balakrishna, K. M., \& Naik, K. G. (2017). Deposition of undoped and Al doped ZnO thin films using RF magnetron sputtering and study of their structural, optical and electrical properties.

Callister, W. D., \& Rethwisch, D. G. (2011). Materials science and engineering (Vol. 5): John Wiley \& Sons NY.

Cruz, N. C. d. (1999). Propriedades estruturais e opticas de filmes finos de oxido de titanio depositados por PECVD.

Floriano, E. A., Scalvi, L. V. A., \& Sambrano, J. R. (2009). Determination of the band energy diagram and absorption fundamental edge in $\mathrm{SnO} 2$, deposited via sol-gel, on quartz. Cerâmica, 55(333), 88-93. 
Ganesh, V. A., Nair, A. S., Raut, H. K., Walsh, T. M., \& Ramakrishna, S. (2012). Photocatalytic superhydrophilic TiO 2 coating on glass by electrospinning. Rsc Advances, 2(5), 2067-2072.

Kasap, S., \& Capper, P. (2017). Springer handbook of electronic and photonic materials: Springer.

Khedmi, N., Rabeh, M. B., \& Kanzari, M. (2014). Thickness Dependent Structural and Optical Properties of Vacuum Evaporated Culn5S8 Thin Films. Energy Procedia, 44, 61-68.

Lin, H.-J., Yang, T.-S., Hsi, C.-S., Wang, M.-C., \& Lee, K.-C. (2014). Optical and photocatalytic properties of Fe 3+-doped TiO 2 thin films prepared by a sol-gel spin coating. Ceramics International, 40(7), 10633-10640.

Liu, G., Liu, A., Zhu, H., Shin, B., Fortunato, E., Martins, R., . . Shan, F. (2015). Low-Temperature, Nontoxic Water-Induced Metal-Oxide Thin Films and Their Application in Thin-Film Transistors. Advanced Functional Materials, 25(17), 2564-2572.

Pazim, R. C. (2011). Caracterização óptica de filmes finos. Programa de Pós-Graduação em Física.

Pehlivan, E., Tepehan, F. Z., \& Tepehan, G. G. (2005). Effect of TiO 2 mixtures on the optical, structural and electrochromic properties of $\mathrm{Nb} 2 \mathrm{O} 5$ thin films. Solar energy materials and solar cells, 87(1), 317-322.

Ruppert, C., Aslan, O. B., \& Heinz, T. F. (2014). Optical properties and band gap of single-and fewlayer MoTe2 crystals. Nano letters, 14(11), 6231-6236.

Santos-Cruz, J., Torres-Delgado, G., Castanedo-Perez, R., Jiménez-Sandoval, S., Jiménez-Sandoval, O., Zuniga-Romero, C. I., . . Z Zelaya-Angel, O. (2005). Dependence of electrical and optical properties of sol-gel prepared undoped cadmium oxide thin films on annealing temperature. Thin Solid Films, 493(1), 83-87.

Scapin, V. d. O. (2004). Aplicação da fluorescência de raios X (WDXRF): Determinação da espessura e composição química de filmes finos.

Serway, R. A., \& John Jr, W. (2010). PRINCÍPIOS DE FÍSICA-ÓPTICA E FÍSICA MODERNA: Cengage Learning Edições Ltda.

Siebentritt, S. (2017). Basics of Chalcogenide Thin Film Solar Cells. Photovoltaic Solar Energy: From Fundamentals to Applications, 169.

Wang, M. C., Lin, H. J., \& Yang, T. S. (2009). Characteristics and optical properties of iron ion (Fe $3+)$-doped titanium oxide thin films prepared by a sol-gel spin coating. Journal of Alloys and Compounds, 473(1), 394-400. 